\title{
TEM Observation on Single Defect in SiC
}

\author{
J. Q. Liu*, M. Skowronski*, P. G. Neudeck**, J. A. Powell** \\ *Department of Materials Science and Engineering, Carnegie Mellon University, \\ Pittsburgh, PA 15213 USA \\ **NASA Glenn Research Center, 21000 Brookpark Road, M.S. 77-1, Cleveland, OH 44135 USA
}

Silicon carbide ( $\mathrm{SiC}$ ) is a wide bandgap semiconductor with outstanding properties, such as high thermal conductivity, high electric breakdown voltage, and high carrier saturation velocity, making it an attractive candidate for potential applications in high-power, high-temperature and highfrequency electronic devices. As an IV-IV compound semiconductor, the crystal structure of SiC can be described in terms of an assembly of corner-sharing tetrahedra. In each tetrahedron, an atom of carbon (or silicon) at the centroid is bonded to four atoms of silicon (or carbon) at the corners. SiC exhibits a number of structural variants called polytypes. The most common polytypes of $\mathrm{SiC}$ are $6 \mathrm{H}, 4 \mathrm{H}, 3 \mathrm{C}$ and 15R (Ramsdell's notation) with $\mathrm{C}, \mathrm{H}$, and $\mathrm{R}$ representing cubic, hexagonal and rhombohedral structure, respectively, and the numbers denoting the periodicity of tetrahedral along c-axis of the crystal. The bandgap of $\mathrm{SiC}$ increases in proportion to its "hexagonality" (from $2.2 \mathrm{eV}$ for $3 \mathrm{C}$ to $3.2 \mathrm{eV}$ for $2 \mathrm{H}$ ). In the past two decades, the technology for the $\mathrm{SiC}$ crystal growth has been improved, including bulk growth, epitaxial growth and heteroepitaxial growth. The investigations of defects in the crystals and electronic devices are indeed of fundamental importance, since the presence of defects affects the crystal physical properties and performance of devices. A recently reported degradation phenomenon, that occurred in $4 \mathrm{H}$ - and $6 \mathrm{H}-\mathrm{SiC}$ diodes during the forward-bias operation, was proposed to be associated with the presence of extended defects in the epitaxial layers.[1-3]

This paper presents one example of extended defect characterizations in $\mathrm{SiC}$ by transmission electron microscopy (TEM). 3C-SiC films were deposited on device-sized mesas fabricated on hexagonal 4H-SiC on-axis substrates.[4] Some 3C-SiC heterofilms are completely free of doubleposition-boundaries (DPB's) and stacking faults (SF's). However, isolated stacking faults were revealed by thermal oxidation on some nearly ideal mesas.[4] We are attempting to determine the detailed microstructure of an isolated fault at its nucleation site by high-resolution TEM. In spite of the small size $(0.1 \times 0.1 \mathrm{~mm})$ of the mesa, the cross-sectional TEM sample was made using a "sandwich" method. Figure 1 illustrates the sample preparation procedures. A conventional TEM image of the fault is shown in Figure 2. High-resolution TEM examination is ongoing.

\section{References}

[1] H. Lendenmann et al, Mater. Sci. Forum 353-356, 727 (2001).

[2] J. P. Bergman et al., Mater. Sci. Forum 353-356, 299 (2001).

[3] J. Q. Liu et al., Appl, Phys. Lett. 80(5), 749 (2002).

[4]. P. G. Neudeck et al., Int. Conf. Silicon Carbide \& Related Mat. 2002 Tech. Dig., p. 730 (2001), to appear in Mat. Sci. Forum (2002). 


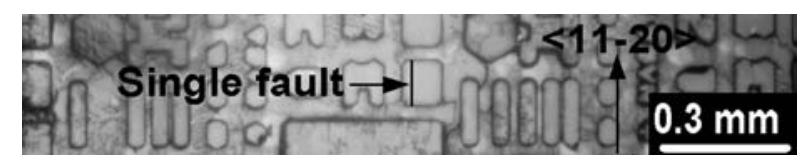

(a)

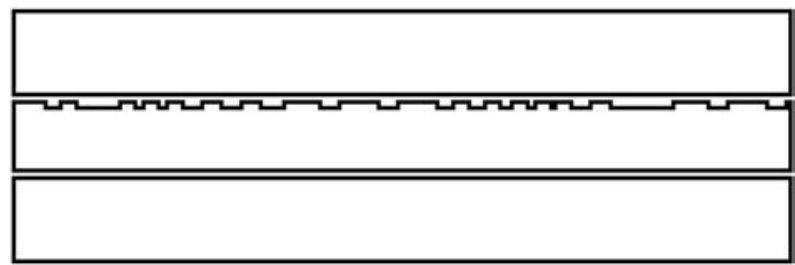

(b)

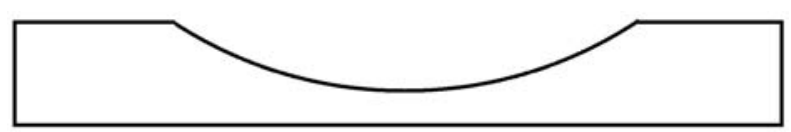

(c)

Figure 1. TEM sample preparation procedures. An optical photo of the top of the sample (a), crosssectional view of a "sandwich" made using M-bond 610 adhesive (b), and final thickness of approximately $20 \mu \mathrm{m}$ was obtained by dimpling (c).

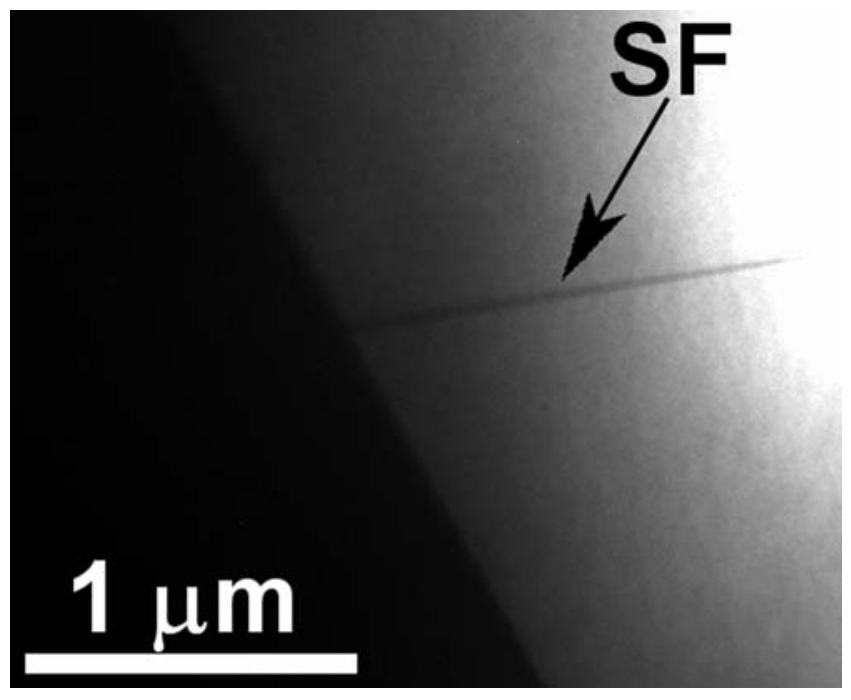

Figure 2. Conventional TEM image of the single stacking fault in 3C-SiC films. 